\title{
Thermophysical investigations of nanotechnological insulation materials
}

Ákos Lakatos

Citation: AIP Conference Proceedings 1866, 030003 (2017); doi: 10.1063/1.4994479

View online: http://dx.doi.org/10.1063/1.4994479

View Table of Contents: http://aip.scitation.org/toc/apc/1866/1

Published by the American Institute of Physics 


\title{
Thermophysical Investigations of Nanotechnological Insulation Materials
}

\author{
Ákos Lakatos ${ }^{1, a)}$ \\ ${ }^{1}$ University of Debrecen, Faculty of Engineering, Department of Building Services and Buildings and Engineering, \\ Ótemetö utca 2-4, 4028 Debrecen, Hungary \\ ${ }^{\text {a)} C o r r e s p o n d i n g ~ a u t h o r: ~ a l a k a t o s @ e n g . u n i d e b . h u ~}$
}

\begin{abstract}
Nowadays, to sufficiently reduce the heat loss through the wall structures with the so-called traditional insulations (polystyrene and fibrous slabs), huge thicknesses $(20-25 \mathrm{~cm})$ must be applied. In some cases there is no place for their applications e.g.: historical or heritage builfings, since the use of nano-insulation materials (aerogel, vacuum ceramic paints) takes place. They are said to be much more efficient insulations than the above mentioned ones, since they should be used in thinner forms. In this article the thermal insulating capability of solid brick wall covered with a silica-aerogel slab with $1.3 \mathrm{~cm}$, moreover with a vacuum ceramic hollow contained paint with $2 \mathrm{~mm}$ thick are investigated. As well as a literature review about the thermal conductivity of nano-technological insulation materials will be given. Comparison of the atomic and thermal diffusion will be also presented.
\end{abstract}

\section{INTRODUCTION}

In the European Union, buildings account for about $20-40 \%$ of the total energy consumption [1-6]. This energy consumption contributes to producing around $25-30 \%$ of $\mathrm{CO}_{2}$ emissions. The reduction both of the energy consumption and the emission of the green house gases can be easily achieved by thermal insulation. Several insulation materials are available, but the use of the nano-technological ones with thin applications is truly spread over. The application of the aerogel is said to be a state-of-the-art thermal insulation solution, and looks to be the most reasonable with the highest potential. Aerogel blankets/panels have already been used at all parts of the building envelopes except by the moisture loaded parts. Commercially available and affordable state-of-the-art aerogels have been reported to have thermal conductivities between $0.014 \mathrm{~W} /(\mathrm{mK})$ and $0.022 \mathrm{~W} /(\mathrm{mK})$ at ambient pressure. However, its high production costs go against its applications. Aerogels have relatively high compression strength, but they are very fragile due to their very low tensile strength, moreover, the blanket/fibrous one is very dusty. A very interesting aspect with aerogels is that they can be produced as either opaque (in a blanket), translucent or transparent materials, thus enabling a wide range of possible building applications [1-7]. Some other articles speak about the efficient use of Vacuum Insulation Panels and others made report on the good insulation properties of hollow nanosphere contaminated vacuum insulation paints. VIP has initial center-of-panel thermal conductivity of $0.004 \mathrm{~W} /(\mathrm{mK})$ or lower, and integration of VIP in building envelopes can reduce $\mathrm{CO} 2$ emissions and contribute towards 'net-zero' or 'near-net-zero' building constructions. Vacuum contaminated nano-ceramic thermal insulation (VCHNS) is sad to be to the most promising thermal insulations. In this article measurements will be presented in order to show the good thermal properties of these materials [14-18]. In Fig. 1 one can see the proposed thermal conductivities of the commonly used insulation materials. 


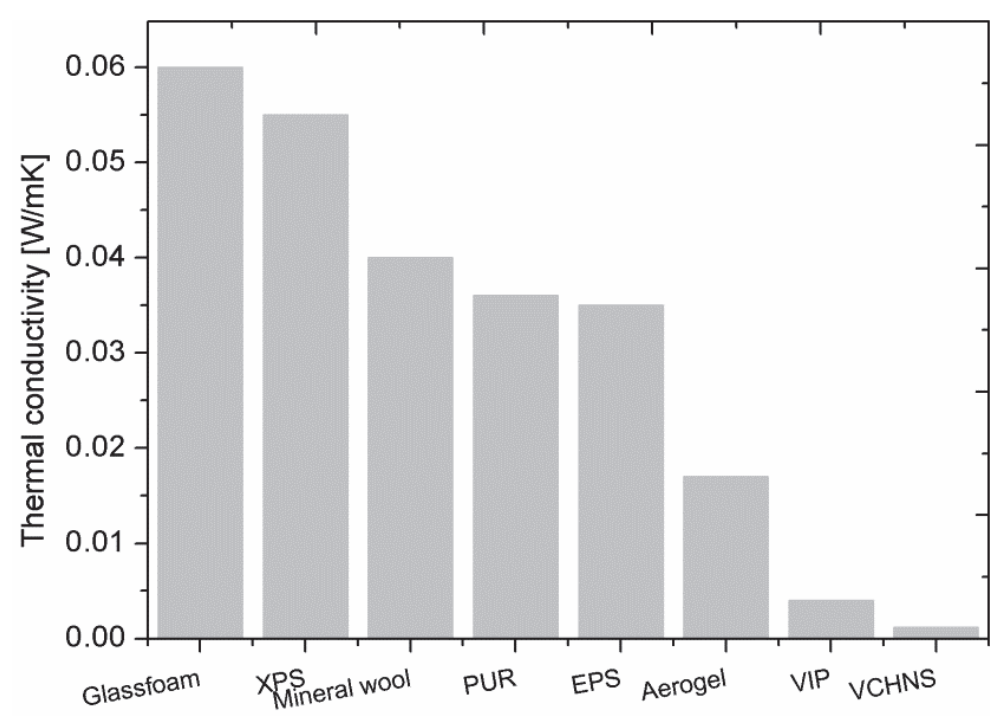

FIGURE 1. The proposed thermal conductivities of the commonly used insulation materials

\section{THE THERMAL CONDUCTIVITY}

The thermal conductivity $\lambda$ of homogenous solid materials can be defined easily by the measurement of the equilibrium heat flow passing through the material resulted by the $\mathrm{dT} / \mathrm{dx}$ temperature gradient:

$$
q=\lambda \frac{\partial T}{\partial x}
$$

where $q$ is thermal energy flow. From this equation one can see that the transport of the heat in a material is a stochastic (randomized) process. It means that the way of the energy through the material is not linear, but it diffuses through the sample suffering collisions. It can easily accepted by the understanding of the meaning of the temperature gradient. For the thermal conductivity of the materials from the kinetic gas-model the following equation can be reached:

$$
\lambda=\frac{1}{3} C \times u \times \Lambda
$$

where $\mathrm{C}$ is the specific heat capacity, $\mathrm{u}$ is the average velocity of the particles and $\Lambda$ is the average free path. This equation was first presented by Debye applied for solid dielectrics. Where $\mathrm{C}$ is the specific heat capacity of the phonons (collective crystal vibrations), $\mathrm{u}$ is the speed of the sound and $\Lambda$ is the average free path of the phonons. (in solid crystals $10^{-8}-10^{-9} \mathrm{~m}$ ).

\section{THE EFFECTIVE THERMAL CONDUCTIVITY}

We cannot apply the above mentioned theory for bulk, regular insulation materials with $10^{1}-10^{-2} \mathrm{~m}$ thicknesses, because we cannot speak about homogenous materials in those cases, resulted by this new definition for thermal conductivity should be given:

$$
\lambda_{\text {eff }}=\lambda_{c, s}+\lambda_{c, g}+\lambda_{r}+\lambda_{\text {conv }}+\lambda_{\text {coupling }}+\lambda_{\text {leak }}
$$

where $\left(\lambda_{c, g}\right),\left(\lambda_{r}\right),\left(\lambda_{c, s}\right)$, and $\left(\lambda_{c o n v}\right)$ are the conductive part of the gas filling, and the solid material, the radition part and the convective part of the gas filling (if there is enough place). Moreover the $\lambda_{\text {coupling }}$ and $\lambda_{\text {leak }}$ can be defined as the followings: $\lambda_{\text {coupling }}=$ thermal conductivity term accounting for second order effects between the various thermal conductivities in Eq.(3), $\lambda_{\text {leak }}=$ leakage thermal conductivity. In order to reach a thermal conductivity as low as possible, each of the above thermal contributions have to be minimized. Normally, the leakage thermal conductivity 
$\lambda_{\text {leak }}$, representing an air and moisture leakage driven by a pressure difference, is not considered as insulation materials and solutions are supposed to be without any holes enabling such a thermal leakage transport. The coupling term $\lambda_{\text {lcoupling }}$ can be included to account for second order effects between the various thermal conductivities in Eq.(3). This coupling effect can be quite complex and will be neglected in the rest of this article. This $\lambda_{\text {eff }}$ is called to effective thermal conductivity. [11]

For bulk materials containing other particles (hollow nanospheres) the flow of the heat can be explained through the following easy sketch (see Fig. 2).

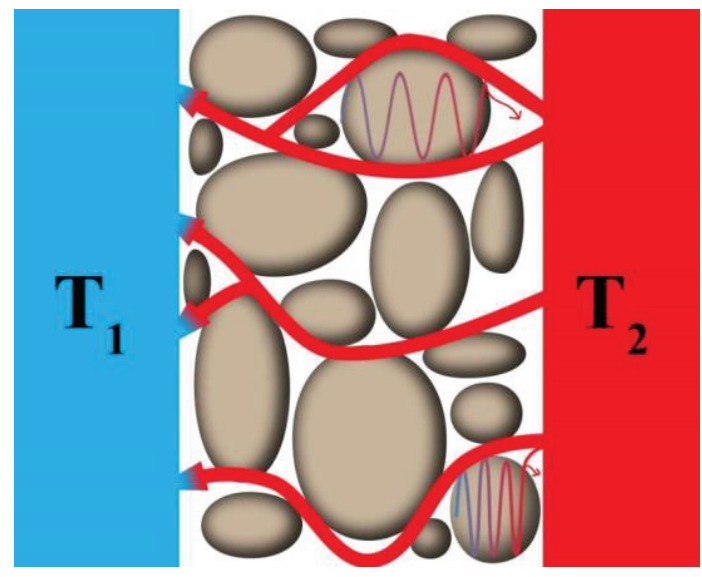

FIGURE 2. The transport of the heat through the inhomogeneous bulk materials [20]

One can see that the heat will always flow through the easiest paths in our case through the added gluing material, while the down slowing effect of the hollows will be negligible.

\section{ANALOGIES AMONG THE TERMAL AND ATOMIC FLOWING PROCESSES}

\section{The Diffusion of Atoms}

In this section the comparison of the mathematics of the atomic diffusion (nano-diffsuion) with the thermal diffusion will be presented. In order to fully understand the atomic flux, diffusion must first be defined. Diffusion is caused by random molecular motion that leads to complete mixing. It follows then that flux can be described as "the rate per unit area at which mass moves. [15] Numerical calculations were done to predict the thermal diffusivity of the materials and the time lag. If we represent the Onsager equation as Eq.(4):

$$
J=D \times \operatorname{grad}(X)
$$

where $J$ is the flux of an extensive property (eg.: atomic flux, heat flux, etc.) and $X$ is the intensive physical property (eg.: atomic concentration, temperature). By using Eq.(4) we can make a connection between the first (Eq.(5)) and the second law of Fick (Eq.(6)) with the thermal conductivity equations (Eqs.(8) (Fourier) and (9)):

$$
J_{A}=-D_{A} \times \operatorname{grad}(C)
$$

where $J_{A}$ is the diffusion flux of the atoms in a given substance, $D_{A}$ is the atomic diffusion coefficient and $\operatorname{grad} C$ is the concentration gradient of the diffusing atoms. Representing the 2nd law of Fick in one dimension and assuming that there are no sources and $D_{A}$ is constant, the following equation can be reached:

$$
\frac{\partial C}{\partial t}=D_{A} \times \frac{\partial^{2} C}{\partial x^{2}}
$$

where $t$ is the time [15]. 


\section{The Thermal Diffusion}

If we represent now the main equation of the thermal conduction which is the modified Fourier's law, a form of the equation is similar to the above mentioned ones (Eqs.(4) and (5)) can be found:

$$
J_{q}=-D_{T} \times \operatorname{grad}(T)
$$

where $J_{q}$ is the heat flux and $\operatorname{grad} T$ is the temperature $(T)$ gradient, however,

$$
D_{T}=\frac{\lambda_{e f f}}{\rho \times c_{p}}
$$

is the thermal diffusion coefficient, $\lambda_{\text {eff }}$ is the above mentioned thermal conductivity, $\rho$ is the mass density and $c_{p}$ is the specific heat of the material. If we represent the modified Fourier's law (Eq.(9)) in one dimension the following equation can be reached by using the following assumptions: the sample is free from heat sources and $D_{T}$ is constant:

$$
\frac{\partial T}{\partial t}=D_{T} \times \frac{\partial^{2} T}{\partial x^{2}}
$$

\section{Calculation Methods for the Retardation Time (Time Lag)}

There are some cases, when the atomic diffusion between pure two A and B materials cannot be represented as a Gaussian function, since the concentration profiles have a complementary error function dependence on depth near the interface and can be modelled as Eq.(4)

$$
C(z, t)=\frac{C_{0}}{2} \times \operatorname{erfc}\left(\frac{z}{2 \times \sqrt{D_{A} t}}\right)
$$

If one is measuring the rate of flow of a gas through a membrane in which the gas dissolves there will be an interval from the moment the gas comes into contact with the membrane until it emerges at a constant rate at the other side. In atomic diffusion is called to delay, time lag or retardation time of the diffusion barrier.

From Eq. (10) the $t$ (in $\mathrm{h}$ ) as the time lag can be reached as:

$$
t(h)=\frac{1}{4 \times \text { Const }^{2} \times D_{A}}: 3600
$$

Similarly to Eq.(10) and (11) the temperature profiles can also be modelled as a complementary error function:

$$
T(z, t)=\frac{T_{0}}{2} \times \operatorname{erfc}\left(\frac{z}{2 \times \sqrt{D_{T} t}}\right)
$$

From Eq.(12) the $t$ (in $\mathrm{h}$ ) as the time lag can be reached as:

$$
t(h)=\frac{1}{4 \times \text { Const }^{2} \times D_{T}}: 3600
$$

Here has to be mentioned that Eqs.(10) and (11) are well known equations for the diffusion profiles in nanodiffusion and it was presented earlier that they can be applied with good approximations for thermal diffusions [15].

\section{COMPARISON OF THE THERMAL CONDUCTIVITIES OF THE "NANOTECHNOLOGICAL" INSULATION MATERIALS}

In our previous papers $[13,15,19,21]$ the measurements of the thermal conductivities of the aerogel insulations with $1.3 \mathrm{~cm}$ and vacuum hollow spheres contaminated insulation paint with $2 \mathrm{~mm}$ thickness applied on a brick based wall with hot box method, moreover their measurements with Holometrix Lambda 2000 apparatus were presented 
also. The measurement order and details can be found clearly in $\operatorname{Ref}[13,15,19,20]$. In those articles the following results were presented (see Fig. 3).

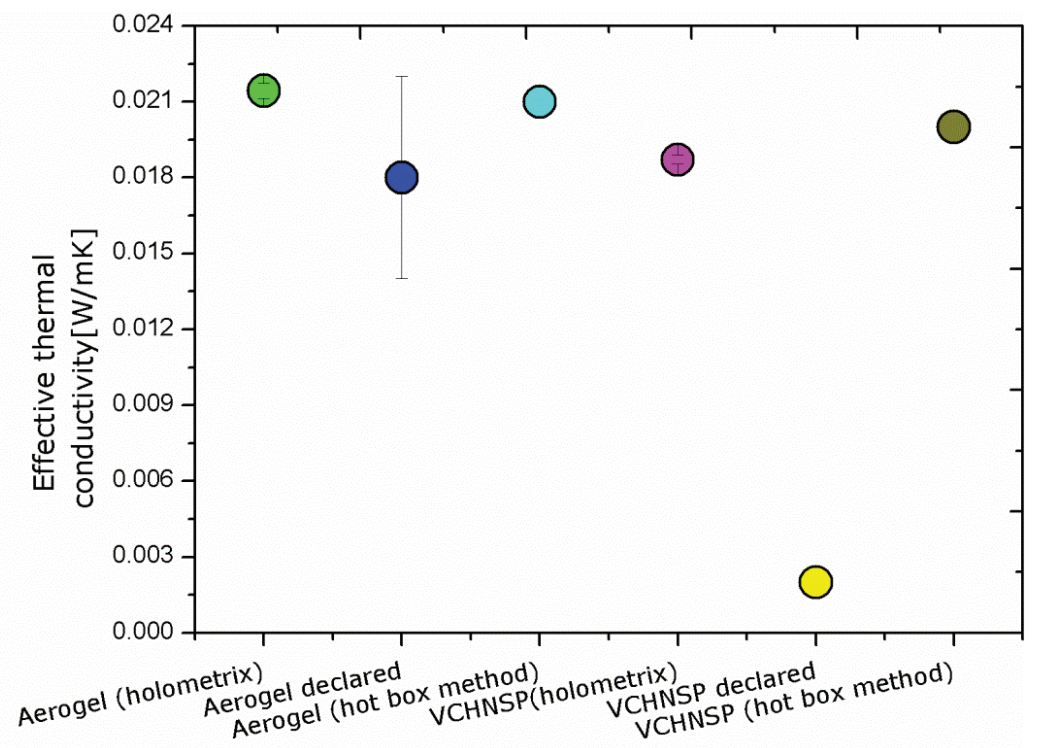

FIGURE 3. The thermal conductivities of the nano-technological insulation materials $[13,15,19,20]$

From Fig. 3 one can see that the results reached both from the measurements with Holometrix apparatus and with calibrated chamber method gave nearly the same values, besides those the declared values are presented too. One the graph one can see that the blue circle has a relatively wide interval. The reason for this that in the literature the value of the thermal conductivity of the aerogel is ranging between $0.014 \mathrm{~W} /(\mathrm{mK})$ and $0.022 \mathrm{~W} /(\mathrm{mK})$. The declared value of the hollow nanospheres is indicated with yellow circle showing very-very low thermal conductivity, however the measurement result is far from this value.

\section{CONCLUSIONS}

Energy conscious building design consists in controlling the thermophysical characteristics of the building envelope such as, firstly, thermal transmittance (U-value), calculated from the thermal conductivities. However, besides the U-value, the envelope thermal inertia (time lag) should also be considered. Besides the laboratory measurements of the building and structural materials, calculations, predictions, modelling and simulations are also important when we design buildings.

Firstly in this article theoretical review for the thermal conductivity and for the analogizes of thermal and atomic diffusion is presented. It was showed that the mathematical equations regarding to the atomic diffusion in "nano" size can be applied for thermal diffusion also.

Secondly, the measurement results of the thermal conductivities of nano-technological insulation materials are presented and their values were compared to the declared values. Here has to be mentioned that these materials are usually called to nano-technological insulation materials, however it does not mean that they can be applied in nanmetric size (thickness), but better implies the name to their manufacturing processes.

\section{ACKNOWLEDGMENTS}

This paper was supported by the János Bolyai Research Scholarship of the Hungarian Academy of Sciences.

\section{REFERENCES}

1. L. Perez-Lombard, J. Ortiz, and C. A. Pout, Energy Build. 40, 394-398 (2008). 
2. A. Borodinecs and A. Kreslins, "Reduction of Cooling and Heating Loads Using Building Envelopes with Controlled Thermal Resistance," in Proceedings of Conference: Air Conditioning and the Low Carbon Cooling Challenge (Network for Comfort and Energy Use in Buildings, London, 2008), pp. 1-7.

3. F. Kalmár and T. Kalmár, Journal of Harbin Institute of Technology (New Series) 14, 81-84 (2007).

4. F. Kalmár, Advanced Materials Research 899, 30-35 (2014).

5. F. Kalmár, "Energy Analysis of Building Thermal Insulation," in Proceedings of the 11th Conference for Building Physics (Dresden, 2002), pp.103-112.

6. Z. Verbai, I. Csáky, and F. Kalmár, Energy Build. 135, 1-9 (2017).

7. S. R. Hostler et al., Int. J. Heat Mass Transf. 52, 665-669 (2008).

8. J. M. Schultz, K. I. Jensen, and F. H. Kristiansen, Sol. Energy Mater. Sol. Cells 89 275-285 (2005).

9. R. Galliano et al., Energy Build. 126, 275-286 (2016).

10. K. Ghazi Wakili et al., Energy Procedia 78, 949-954 (2015).

11. B. P. Jelle, Energy Build. 43 2549-2563 (2011).

12. T. Stahl et al., Energy Build. 44, 114-117 (2012).

13. A. Lakatos, Energy Build. 139, 506-516 (2017).

14. A. Lakatos, Advanced Materials Research 1016 733-737 (2014).

15. A. Lakatos, I. Csáky, and F. Kalmár, Mater. Struct. 48, 1343-1353 (2015).

16. K. Song and P. Mukhopadhyaya, Int. Rev. Appl. Sci. Eng. 7, 113-119 (2016).

17. J. Fricke, U. Heinemann, and H. P. Ebert, Vacuum 82, 680-690 (2008).

18. R. G. Ogden, S. Resalati, and C. C. Kendrick, "A Combined Operational and Embodied CO2 Approach: The Limits of Conventional Insulation Materials and Case for High Performance Vacuum Technology," in Proceedings of the 12th International Vacuum Insulation Symposium (IVIS 2015) (Nanjing University of Aeronautics and Astronautics, Nanjing, China, 2015), pp. 131-135.

19. A. Lakatos, Energiagazdálkodás 57, 21-25 (2016).

20. A. Lakatos, "A Theoretical Approach to Estimate the Time Lag of Building Envelopes," in 16th International Multidisciplinary Scientific GeoConference SGEM 2016 (STEF92 Technology Ltd., Vienna, 2016), pp. 403410. 